\title{
Dayside isotropic precipitation of energetic protons
}

\author{
V. A. Sergeev ${ }^{1}$, G. R. Bikkuzina ${ }^{1}$, P. T. Newell ${ }^{2}$ \\ ${ }^{1}$ Institute of Physics, St. Petersburg University, St. Petersburg 198904, Russia \\ ${ }^{2}$ The Johns Hopkins University Applied Physics Laboratory, Laurel, MD 20723-6099, USA
}

Received: 24 September 1996 / Revised: 13 March 1997 / Accepted 27 March 1997

\begin{abstract}
Recently it has been shown that isotropic precipitation of energetic protons on the nightside is caused by a non-adiabatic effect, namely pitch-angle scattering of protons in curved magnetic field lines of the tail current sheet. Here we address the origin of isotropic proton precipitation on the dayside. Computations of proton scattering regions in the magnetopheric models T87, T89 and T95 reveal two regions which contribute to the isotropic precipitation. The first is the region of weak magnetic field in the outer cusp which provides the $1-2^{\circ}$ wide isotropic precipitation on closed field lines in a $\sim 2-3$ hour wide MLT sector centered on noon. A second zone is formed by the scattering on the closed field lines which cross the nightside equatorial region near the magnetopause which provides isotropic precipitation starting $\approx 1.5-2 \mathrm{~h}$ MLT from noon and which joins smoothly the precipitation coming from the tail current sheet. We also analyzed the isotropic proton precipitation using observations of NOAA low altitude polar spacecraft. We find that isotropic precipitation of $>30$ to $>80 \mathrm{keV}$ protons continues around noon forming the continuous oval-shaped region of isotropic precipitation. Part of this region lies on open field lines in the region of cusp-like or mantle precipitation, its equatorward part is observed on closed field lines. Near noon it extends $\sim 1-2^{\circ}$ below the sharp boundary of solar electron fluxes (proxy of the open/closed field line boundary) and equatorward of the cusp-like auroral precipitation. The observed energy dispersion of its equatorward boundary (isotropic boundary) agrees with model predictions of expected particle scattering in the regions of weak and highly curved magnetic field. We also found some disagreement with model computations. We did not observe the predicted split of the isotropic precipitation region into separate nightside and dayside isotropic zones. Also, the oval-like shape of the isotropic boundary has a symmetry line in 10-12 MLT sector, which with increasing activity rotates
\end{abstract}

Correspondence to: V. A. Sergeev, Sergeevl@snoopy.niif.spb.su toward dawn while the latitude of isotropic boundary is decreasing. Our conclusion is that for both dayside and nightside the isotropic boundary location is basically controlled by the magnetospheric magnetic field, and therefore the isotropic boundaries can be used as a tool to probe the magnetospheric configuration in different external conditions and at different activity levels.

\section{Introduction}

A remarkable feature of energetic proton precipitation is their isotropic precipitation (with fluxes being isotropic over the loss cone) which exists at any local time in any disturbance conditions (Hauge and Soraas, 1975; Lundblad et al., 1979) and forms the oval-shaped isotropic precipitation zone (Sergeev and Gvozdevsky, 1995). Recently this precipitation attracted much attention because of its potential importance for evaluation of magnetotail magnetic configuration. As has been shown by Sergeev et al. (1993) and Sergeev and Gvozdevsky (1995), on the nightside this isotropic precipitation is formed by particle pitch-angle scattering in the tail plasma sheet where the adiabatic motion is violated in the weak and curved magnetic field regions. This ever present and effective scattering mechanism provides the isotropic precipitation if the ratio of magnetic field curvature radius $\left(R_{c}\right)$ to the proton gyroradius $(\rho)$ is $<8$ (see also Delcourt et al., 1996). This threshold value corresponds to the boundary between adiabatic and weekly chaotic regimes of particle motion, which is seen at low altitudes as the equatorward boundary of isotropic proton precipitation (isotropic boundary, or IB). A number of observational characteristics (the energy dependence of isotropic boundary etc.), a high correlation (correlation coefficient $\sim 0.9$ ) between the IB latitude and magnetic field 
inclination at geosynchronous orbit as well as good quantitative agreement between predicted and observed positions of isotropic boundaries all confirm this mechanism of isotropic precipitation on the nightside (Sergeev et al., 1993, Sergeev and Gvozdevsky, 1995).

The origin of the isotropic proton precipitation on the dayside is not as clear as for the nightside. The main difficulty concerns the complicated shape of the magnetic field lines in the proximity of magnetic cusp. There are two potential regions of weak magnetic field which may provide the conditions for nonadiabatic scattering of protons and, therefore, provide their isotropic precipitation. First, there is a weak magnetic field in the high-latitude high-altitude cusp region near the magnetopause (referred to hereafter as the 'outer cusp'), where the magnetosheath plasma flows along the magnetopause surface and does not require substantial magnetospheric magnetic field to balance the impulse carried by plasma. The effectiveness of ion scattering in that region was recently confirmed in trajectory computations for lower energy $(<10 \mathrm{keV})$ protons (Alem and Delcourt, 1995), it was also discussed by Lyons et al. (1994). The authors of both papers also presented the examples of observations to show that isotropic precipitation of energetic protons extends equatorwards of the equatorward boundary of cusp-like precipitation, and argue that it lies on closed field lines. However, it is unclear from these computations what is the longitudinal extent of the so produced isotropic precipitation, how it joins the isotropic precipitation from magnetotail, and what is the latitudinal extent of that dayside precipitation for higher energy (tens to hundred $\mathrm{keV}$ ) protons.

The question of longitudinal extent of near-cusp precipitation is especially important in view of another region of weak magnetic field strength on closed field lines situated in the equatorial region near the nightside part of magnetopause. According to the magnetospheric mapping in the Tsyganenko 1989 model (Tsyganenko, 1989) performed by Kaufmann et al. (1993) this region is also mapped into the ionosphere along the magnetic field lines very close to the noon. For example, in their Fig. 1 and 4, the ionospheric footpoints of equatorial points displaced from magnetopause inward by $0.1 R_{e}$ extend in local time from the noon only by 1.5-2 h. However, as mentioned by Kaufmann et al., (1993) the mapping in this region using the T89 model is not very reliable because that model does not include sharp predetermined magnetopause. Nevertheless it shows that equatorial near-magnetopause scattering region is also a possible candidate to provide dayside isotropic precipitation.

When dealing with isotropic precipitation we must not forgot the possibility that isotropic precipitation may be caused by plasma turbulence which may be strong enough in both these regions. To distinguish this mechanism from nonadiabatic scattering in a curved weak magnetic field (which we term 'regular scattering' to distinguish it from 'turbulent scattering'), one may (1) use the predictions of scattering based on magnetic field models and (2) check a specific feature of scattering in weak regular magnetic field, namely, the energy-dependent position of isotropic boundary (it should be located at lower latitude for protons of larger energy and gyroradius).

The purpose of this work is to study quantitatively the role of regular scattering in providing the isotropic proton precipitation near the noon. In Sect. 2 we compute the regular scattering regions and their ionospheric footpoints for a number of magnetospheric models (T87, T89 and T95). These model predictions are compared with observations presented in Sect. 3, dayside isotropic precipitation of energetic protons based on data from TIROS and NOAA-6,-7,-10 low-altitude spacecraft are studied. We consider the characteristics of isotropic precipitation, position of isotropic boundary with respect to the cusp location and dependence of its position on particle energy. We also investigate the shape of isotropic boundary near noon and its dependence on activity indices and interplanetary parameters. Then, results of modeling and observations are discussed to decide on the possible mechanism of dayside precipitation and some unresolved issues are mentioned.

\section{Regions of regular particle scattering in the magnetosphere and their ionospheric footpoints according to empirical magnetospheric models}

We used three empirical magnetospheric models T87, T89 and T95. Whereas the earlier models, T87 and T89 (Tsyganenko, 1987, 1989), had no predetermined magnetopause, the last model T95 (Tsyganenko, 1996) includes the empirically-established sharp magnetopause where the normal magnetic field component vanished in the absence of interconnection magnetic field (which simulates the IMF penetration into the magnetosphere due to the magnetic reconnection). The computations in the different magnetospheric models are presented for zero dipole tilt angle (Figs. 1-4), but we also briefly comment on the effects of changing dipole tilt (Fig. 5).

Magnetic field lines were traced starting from the ionosphere at the given magnetic meridian and identified the last closed field line. Then we studied a lower latitude with a 0.1 degree step and, after tracing the field line, the point characterized by the minimal value of $R_{c} / \rho$ was found and checked whether the threshold of pitch-angle scattering in curved weak magnetic field was exceeded on that field line. As a criterion of isotropic pitch-angle scattering (over the ionospheric loss cone) we used the same threshold condition, $R_{c} / \rho<8$, which was previously determined in the trajectory simulations (Sergeev et al., 1993; Delcourt et al., 1996).

Examples of magnetic field lines at 12 and 14 MLT meridians in the T89 model are shown in Fig. 1a, b, respectively. The regions of regular pitch-angle scattering are shown by different symbols depending on the position where the scattering takes place (triangles or stars in outer cusp and nightside regions, correspondingly). Unlike the magnetotail, the magnetic field magnitude may have a few extrema (minima and maxima) on the same field line. At the noon meridian 

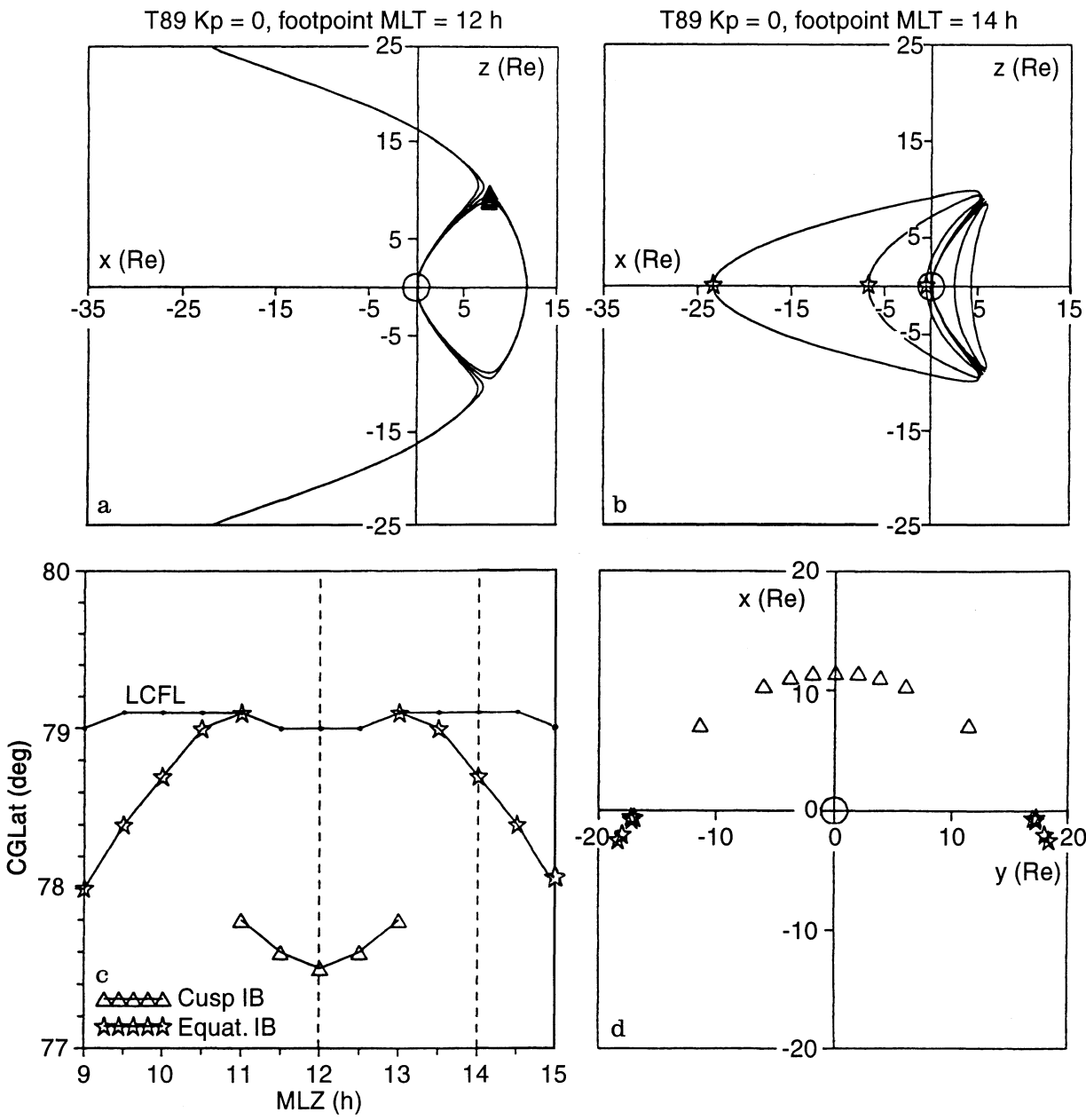

Fig. 1a-d. Results of tracing the magnetic field lines in T89 model with zero dipole tilt angle. Right panels flank views of magnetic field lines started from a $12 \mathrm{~h} \mathrm{MLT}$ or b $14 \mathrm{~h} \mathrm{MLT}$ meridian in the ionosphere with a step $0.1^{\circ}$ in latitude. Regions where the condition of isotropic scattering of 80 $\mathrm{keV}$ protons $\left(R_{c} / \rho<8\right)$ is fulfilled are marked by triangles (or asterisks) for high-latitude cusp region (or nightside equatorial region near the magnetopause, correspondingly). c Ionospheric projections of isotropic boundaries for $80 \mathrm{keV}$ protons scattered in the highlatitude cusp region (triangles) or in the nightside equatorial region (asterisks). Position of the last closed field line ( $L C F L)$ is also shown. d Equatorial mapping of the isotropic boundaries shown on previous plot

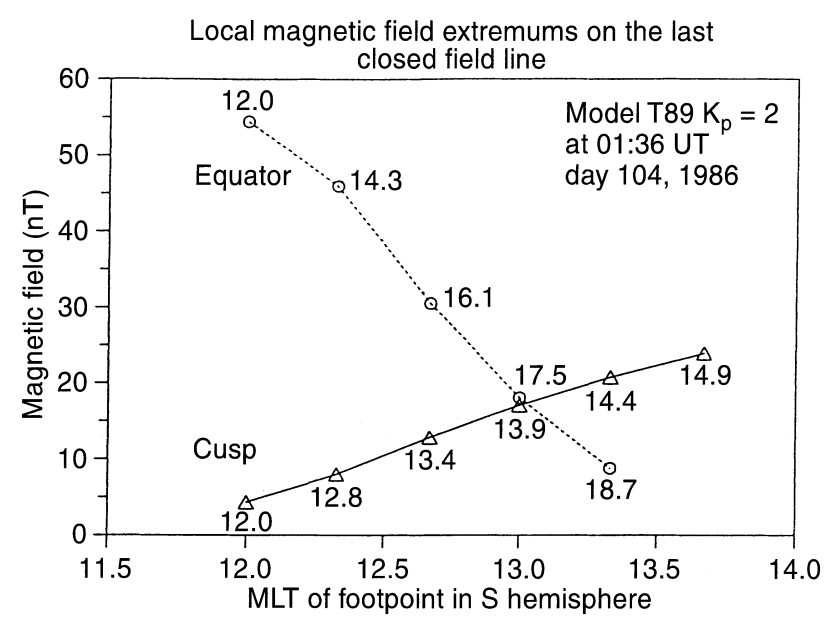

Fig. 2. Magnetic field values in equatorial region (circles) and highlatitude cusp (triangles) on the last closed field line against the local time of its ionospheric footpoint. The points are labelled with actual MLT where the field line crossed the cusp region or equatorial region. (Last closed field line was determined by tracing the field lines starting at fixed MLT meridian with a step $0.1^{\circ}$ in geomagnetic latitude)

(Fig. 1a) the field lines lie in the noon-midnight plane and the isotropic scattering occurs only in the highlatitude (cusp) minimum of the magnetic field. In the equatorial region there is a local maximum of the

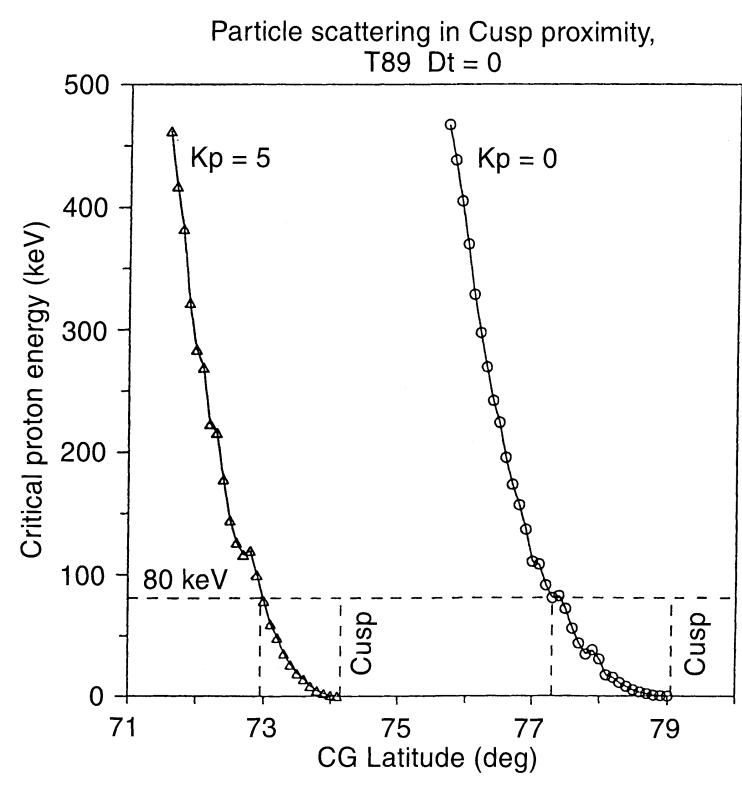

Fig. 3. Positions of isotropic boundary latitude at the noon meridian in T89 model for protons of different energy characterizing the expected energy dispersion of isotropic boundary

magnetic field magnitude (see Fig. 2). Moving away from noon in magnetic local time (14 h MLT in Fig. 1b), the outermost closed field line stays approxi- 


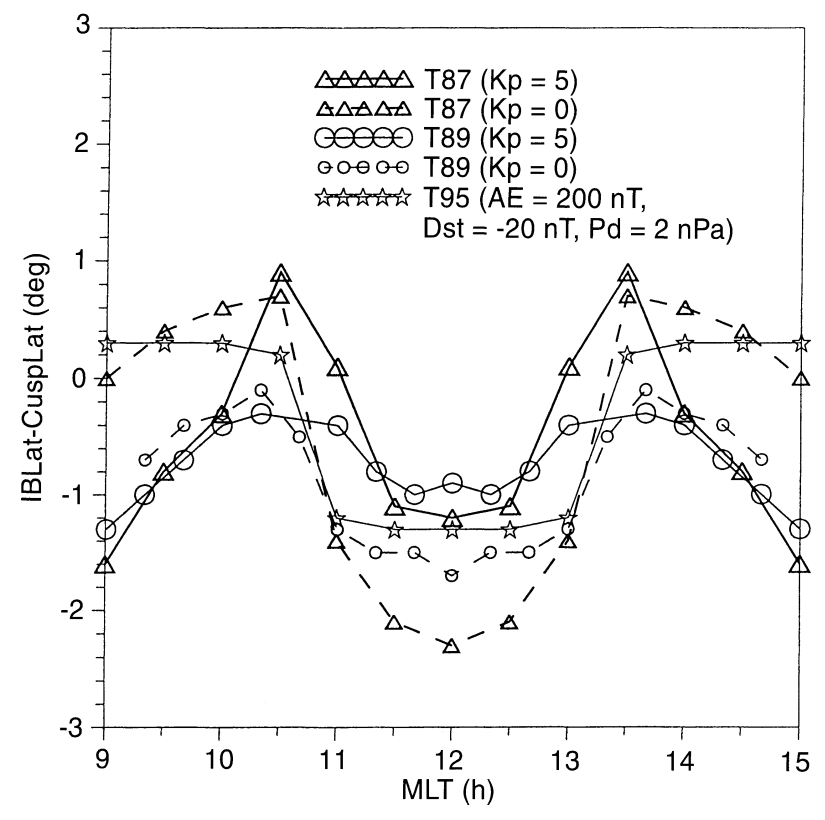

Fig. 4. Shape of dayside isotropic boundary in different models for zero dipole tilt. To facilitate the comparison, all profiles are shifted in latitude so that last close field line has a zero latitude mately in the meridian plane until it reaches the outer cusp latitude (with local B-field minimum still observed there). Then it sharply turns tailward and crosses the equatorial region near the magnetopause where the field minimum is detected.

A comparison of near-cusp and equatorial B-field values on the same field line in Fig. 2 indicates that on the outer closed field lines, the near-cusp magnetic field is the smaller of these two and determines the regular scattering in the $\sim 2 \mathrm{~h}$ MLT wide sector centered on the noon. In this sector our computations (Fig. 1c) predict the isotropic precipitation region which occupies $\sim 1.5^{\circ}$ latitude between the isotropic boundary and the last closed field line (LCF).

According to Fig. 2, more than $1.5 \mathrm{~h}$ MLT later than noon the equatorial magnetic field is smaller and, therefore, determines the scattering efficiency. The regular scattering in the equatorial region first becomes possible at field lines with footpoints at $\sim 11 \mathrm{~h}$ and $\sim 13 \mathrm{~h}$ MLT meridians, and the band width of isotropic precipitation produced in the nightside equatorial region increases with increasing distance from noon. According to Fig. 1c, the isotropic precipitation region should have its minimal width at the distance $\sim 1.5 \mathrm{~h}$ MLT from noon.
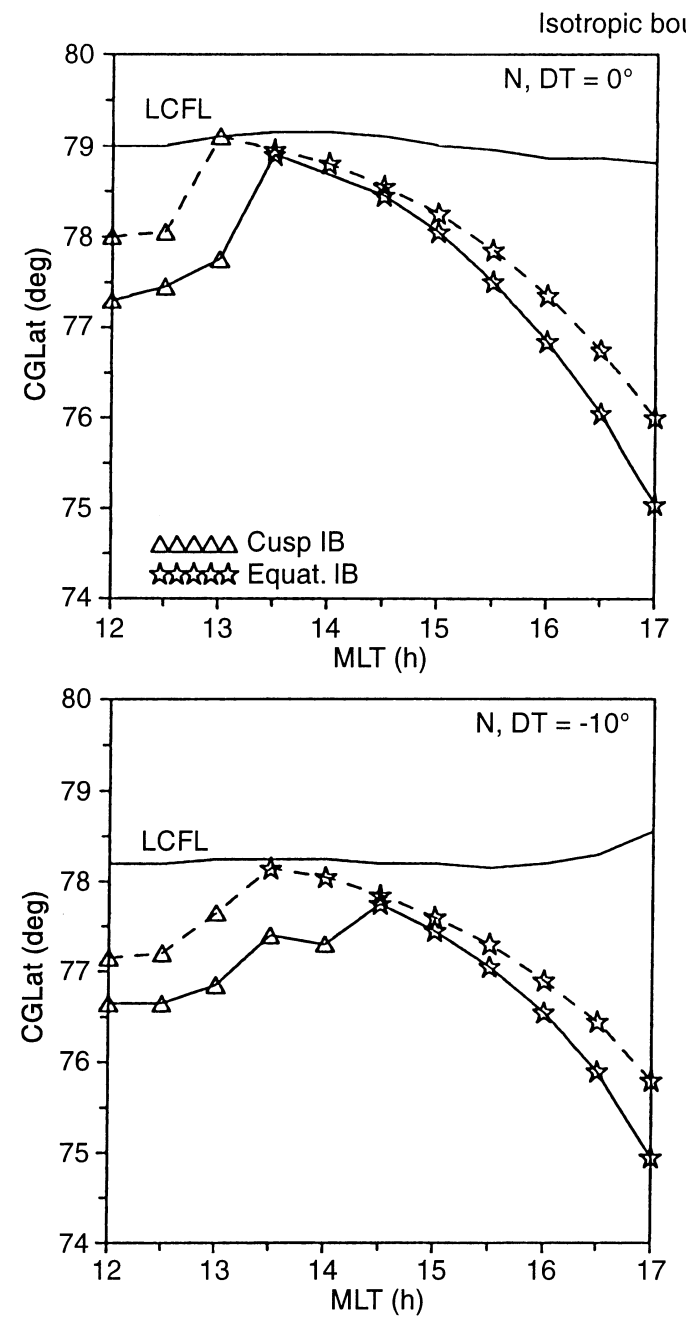
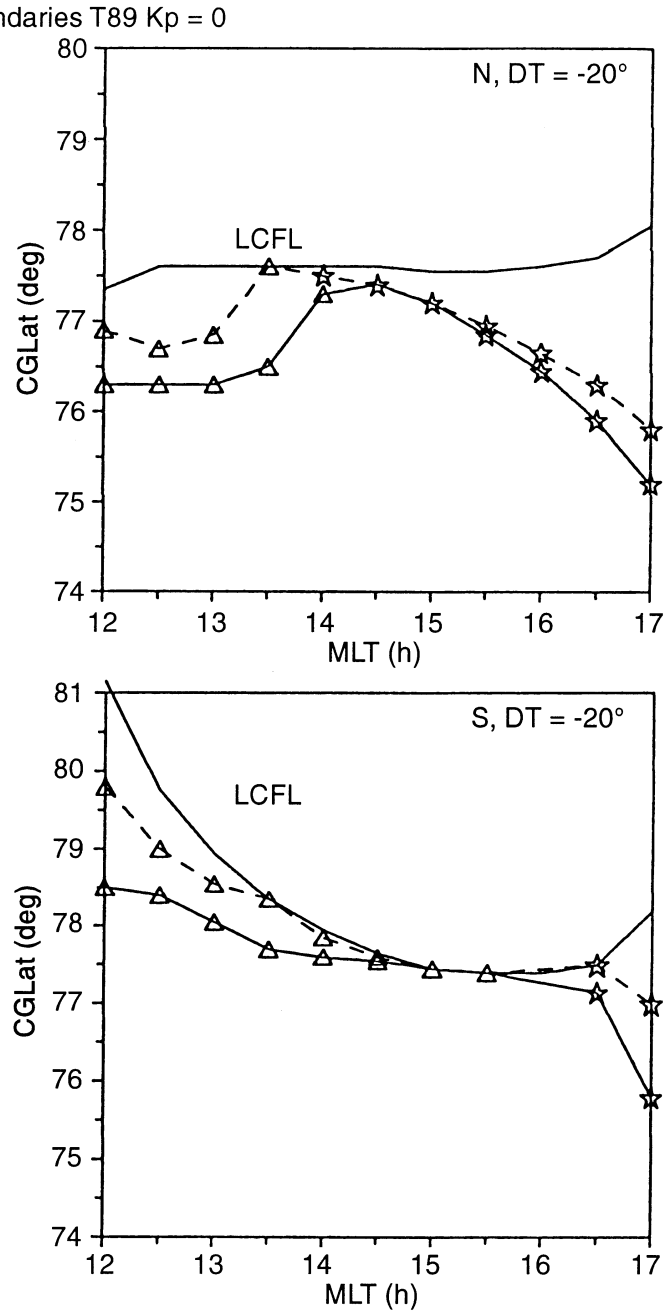

Fig. 5. Ionospheric projections of isotropic boundaries for $30 \mathrm{keV}$ and $80 \mathrm{keV}$ protons scattered in the highlatitude cusp region (triangles) or in the nightside equatorial region (asterisks) for dipole tilt angles $0^{\circ},-10^{\circ}$ and $-20^{\circ}$. Lower left represents the Southern Hemisphere whereas other three panels correspond to the Northern Hemisphere. Only the afternoon sector is shown here 
Figure 3 shows how the width of isotropic precipitation zone at the noon meridian increases with increasing the proton energy. Since this energy dispersion is a specific feature of the regular scattering mechanism, its observational confirmation may help to distinguish this scattering mechanism from other mechanisms, such as turbulent scattering.

Computations with other Kp versions of T89 model, as well as with other models (T87 and T95) show the same systematic behaviour as described. The shape of the isotropic boundary predicted by different models is shown in Fig. 4, some other quantitative information is given in Table 1. This shape is a little different in different models, reflecting the different functions used in different models to describe the contributing current systems. It is flatter in the latest T95 model however the relative changes of the modeled IB latitude are almost the same in each model: all models display the highest IB latitude (and therefore the smallest latitudinal width of isotropic precipitation) at a distance of $\sim 1.5$ hours from the noon position.

A basic difficulty in the comparison between different models was the problem of how to define the boundary between closed and open field lines (LCFL) in prenoon and afternoon MLT sectors. The reason is that the models are valid in a limited range of distances (until about $60-70 R_{e}$ down the tail) and, therefore, the character (open or closed) cannot be determined for the field lines which exit from this domain. That is why we show (Fig. 4) the IB latitudes normalized to the position of the last closed field line at noon, which is reliably determined in all models.

With this difficulty in mind, most of the features described here for the T89 model are qualitatively the same as in other models as well as at different disturbance levels. All models predict the isotropic precipitation region of $80 \mathrm{keV}$ protons extending about $1-2^{\circ}$ latitude below the outer cusp projection at the noon meridian (see Table 1), this width shows a tendency to decrease with increasing disturbance level. Our computations also predict a minimal width of the isotropic precipitation at $\sim 13.5$ and $\sim 10.5 \mathrm{~h}$ MLT meridians (see Fig. 4) as well as the amount of energy dispersion such as that shown in Fig. 3 .

The effects of the dipole tilt can be seen from Fig. 5. For non-zero tilt angle the character of isotropic precipitation is basically the same but there are some quantitative differences. A separation line between precipitation regions originated at noon (cusp) and on the nightside (equatorial region) also exists but it shifts further from noon, from $\sim 13.5 \mathrm{~h}$ at DT $=0$ to $\sim 14$ $14.5 \mathrm{~h}$ MLT at DT $=-20$ in the Northern Hemisphere. Considerable difference exists in the shape of boundaries in the northern (winter) and southern (summer) hemisphere, but the uncertainty in the interpretation of the LCFL already mentioned (at local times other than noon it gives only the low latitude limit of the possible last closed field line) makes sensible comparisons difficult. The latitudinal width of the isotropic precipitation zone at noon also changes, it becomes smaller (larger) in winter (summer) season, but its value is still in the range $1-2^{\circ}$ found from different models for zero dipole tilt (Table 1 and Fig. 4).

When discussing the model predictions we mostly emphasized the region on the closed field lines where a high flux of suprathermal protons always exists in the plasma sheet. The actual width of the dayside isotropic proton precipitation region can be larger because the precipitated energetic protons may appear on the open field lines as well. These particles (originating from magnetosheath or magnetosphere) may either be transported by the poleward convection during their journey between the magnetopause and the ionosphere, or if they appear, for some reason, on open field lines and move through the outer cusp, they may be scattered in the same way in the highly curved and weak magnetic field of the outer cusp. Accordingly, the poleward boundary of isotropic proton precipitation at dayside is expected to be more variable and dependent on both the pattern and intensity of the plasma convection. That is why in the following analysis of observations we concentrate on the study of the equatorward boundary of isotropic ion precipitation (isotropic boundary) which is located on the closed field lines and, presumably, is controlled by the magnetospheric magnetic field configuration.

\section{Observations of dayside isotropic proton precipitation}

\subsection{Instrumentation and examples of isotropic precipitation}

We used data from low-altitude polar-orbiting spacecraft (TIROS, NOAA-6,-7, and -10) which made measurements at $\sim 850 \mathrm{~km}$ altitude with similar instruments on each spacecraft. Typically two sun-synchro-

Table 1. Latitudes of isotropic boundary of $80 \mathrm{keV}$ protons (IB) and of the last closed field line (LCFL) at noon meridian as well as the minimal width of isotropic precipitation (LCFL-IB) in different magnetospheric models

\begin{tabular}{lccccc}
\hline Model & T87 & T87 & T89 & T89 & $\begin{array}{l}\text { T95 } \\
A E=200 \mathrm{nT},\end{array}$ \\
Parameters & $\mathrm{Kp}=0$ & $\mathrm{Kp}=5$ & $\mathrm{Kp}=0$ & $\mathrm{Kp}=5$ & $\begin{array}{l}P_{d}=2 \mathrm{nPa}, \\
\text { Dst }=-20 \mathrm{nT}\end{array}$ \\
\hline LCFL & 79.7 & 72.9 & 79.0 & 74.1 & 78.5 \\
IB & 77.4 & 71.7 & 77.5 & 73.0 & 77.2 \\
LCFL-IB & 2.3 & 1.2 & 1.5 & 0.9 & 1.3 \\
\hline
\end{tabular}


nous spacecraft were in orbit with orbital planes aligned approximately along 03-15 h MLT (TIROS, NOAA-7) and 21-09 h MLT (NOAA-6, -10). Due to peculiarities of their orbits all dayside crossings occur in the Northern Hemisphere. The time resolution of the instruments is $2 \mathrm{~s}$. For more information about the spacecraft and instruments see Hill et al., (1985).

The MEPED instrument measured the energetic particle fluxes in two perpendicular directions, including the precipitating flux (pitch angles in the center of the loss cone at polar latitudes) as well as the locally trapped flux (local pitch angles about $90^{\circ}$ ). In this study we mostly use two proton energy channels, 30-80 keV and 80-250 keV $\left(P_{1}\right.$ and $\left.P_{2}\right)$ as well as $>30 \mathrm{keV}$ electrons (channel $E_{1}$ ) which indicates closed magnetospheric field lines. Another instrument (TED) measured the precipitated flux of auroral protons and electrons in 11 energy channels between $0.3-20 \mathrm{keV}$. Only integral characteristics (integral energy flux and channel number giving the largest contribution to the energy flux) and the energy flux from 4 of 11 channels were received on the ground. Data from this instrument may be used to detect the cusp-like precipitation. However the relatively high low-energy threshold $(0.3 \mathrm{keV})$ and absence of detailed distribution function prevented us from careful identification of the cusp proper, LLBL or mantle plasmas. All these three will be referred to simply as cusp-like precipitation.

Figure $6 \mathrm{a}, \mathrm{b}$ shows spacecraft footpoint trajectories in the Northern Hemisphere during the quiet period on August 12, 1979. NOAA-6 and TIROS footpoints skimmed almost simultaneously along the dayside auroral oval in the afternoon and prenoon sectors, correspondingly, and isotropic proton precipitation was recorded continuously throughout the dayside as indicated by crosses. Figure $6 \mathrm{c}$, d shows particle flux recordings for the first event, the isotropic boundaries and a possible equatorward cusp-like boundary near the noon position is shown by the vertical lines. The latter boundary was defined here based on (1) large increase of $\sim 1 \mathrm{keV}$ proton flux (in T DP-3 channel, upper block), combined with (2) decrease of electron average energy to lowest possible value $(0.3 \mathrm{keV}$, corresponding to channel number 1 in T E-M parameter, second block from the top; (the same identifications of the equatorward cusp boundary were used by Carbary and Meng, 1986).

Two peculiarities are seen in these skimming passes. First, during the observation of isotropic proton precipitation (between 040529 and 041313 UT on NOAA-6 and between 040110 and 040655 UT on TIROS) the locally trapped flux of $30 \mathrm{keV}$ electrons (bottom block) continues to be high. This suggests that the isotropic proton precipitation was recorded on closed field lines. Second, the ratio of precipitated proton flux to the trapped flux may show multiple irregular variations (encountering alternatively isotropic or trapped proton distributions) near the equatorward boundary. The largest temporal drop of precipitated ion flux was recorded by the TIROS spacecraft in the prenoon sector at $\sim 040130$ UT and $\sim 040300$ UT at $\sim 10.9$ and $\sim 09.9 \mathrm{~h}$ MLT respectively (Fig. 6d), they were associated with a drop of $1 \mathrm{keV}$ ion flux (T DP-3), increase of electron energy (T E-M) and with some increase of the trapped flux of high-energy electrons (M $\left.E_{1}\right)$. All these are indicators of temporal entrance to the deeper shells of the magnetosphere. This peculiarity is typical of skimming passes when the spacecraft probably passes almost along the isotropic boundary. This may be due to small undulations of the isotropic boundary or due to the temporal variations of scattering process in that region.

When a spacecraft crosses the dayside region almost along the meridian the picture typically looks simpler. An example of such a crossing between 12 and $13 \mathrm{~h}$ MLT is given in Fig. 7, it took place on the same day a few hours later. The cusp-like precipitation was recorded between 76.2 and $79^{\circ}$ CGLat. Isotropic proton precipitation continued throughout most of this region, however it also extends $\sim 2^{\circ}$ equatorward of the cusp precipitation $\left(74.3^{\circ} \mathrm{CGLat}\right.$ in $P_{1}$ channel), well into the closed field line region. Also, in this example the energy dispersion of the isotropic boundary can be seen clearly with $80 \mathrm{keV}$ proton IB (channel $P_{2}$ ) recorded $\sim 0.3^{\circ}$ equatorward of that in $30 \mathrm{keV}$ protons $\left(P_{1}\right)$. No irregular structures in the precipitated/trapped flux ratio, as in the previous skimming passes, are seen in this traversal across the $\sim 5^{\circ}$ latitude-wide isotropic precipitation.

\subsection{Statistical characteristics of dayside isotropic boundary}

Our primary subject, here is the isotropic boundary (IB) of the energetic protons which is always located on closed field lines, equatorward of the cusp-like precipitation. The first question is whether the isotropic proton precipitation has indentations (regions of small thickness) at $\sim 10.5$ and $13.5 \mathrm{~h} \mathrm{MLT}$ as is predicted by the model computations (Fig. 4).

First, we studied the average behavior of the IB latitude of $30 \mathrm{keV}$ protons as a function of the magnetic local time. To achieve a homogeneous orbital coverage we used the data for a six month period in 1979 (JulyDecember) when data were available from two spacecraft (TIROS and NOAA-6), which had nearly perpendicular orbital planes. There were more than 3000 orbits for this period which crossed the isotropic boundary in

\footnotetext{
Fig. 6a-d. Examples of simultaneous observations by two spacecraft skimming along the dayside oval: a, b spacecraft trajectories for 2 episodes on August 12, 1979. Segments of the orbit with isotropic precipitation of $30 \mathrm{keV}$ protons shown by circles, positions of isotropic boundaries by crosses, the cosine approximation of isotropic boundary shape is also shown by the solid line on each plot c, d Particle characteristics neasured by TIROS and NOAA6 during the first episode (trajectories shown in Fig. 4A). From top to bottom: count rate of $\sim 1 \mathrm{keV}$ proton flux; channel number which gives largest contribution to the energy flux of precipitated electrons (numbers 1 to 11 correspond to energies from $0.3 \mathrm{keV}$ to $20 \mathrm{keV}$ ); count rates of trapped and precipitated fluxes of energetic particles $\left(P_{1}\right.$ and $P_{2}$ corresponds to protons at $>30$ and $>80 \mathrm{keV}$, and $E_{1}$ corresponds to electrons with $E>30 \mathrm{keV}$ )
} 

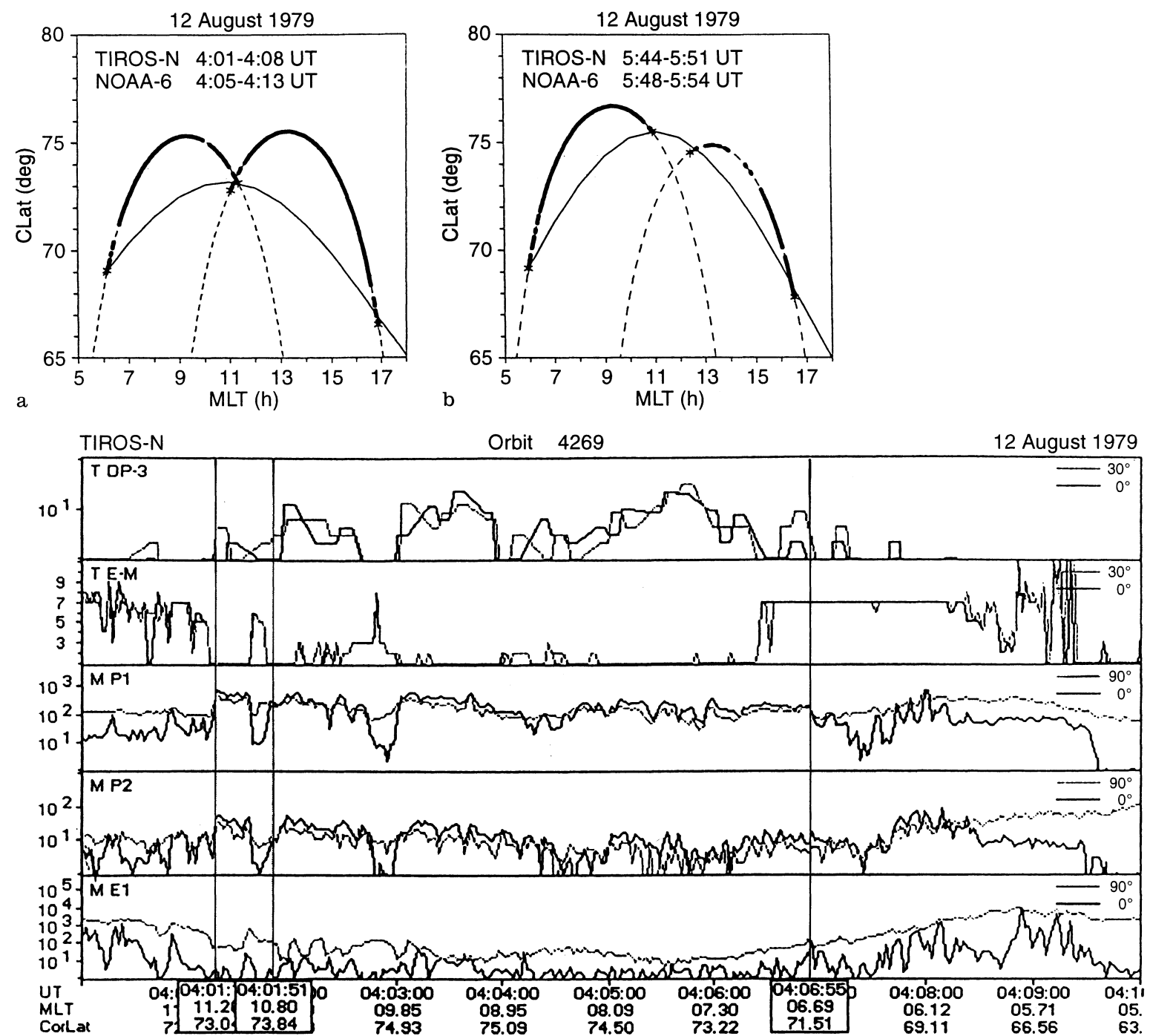

c

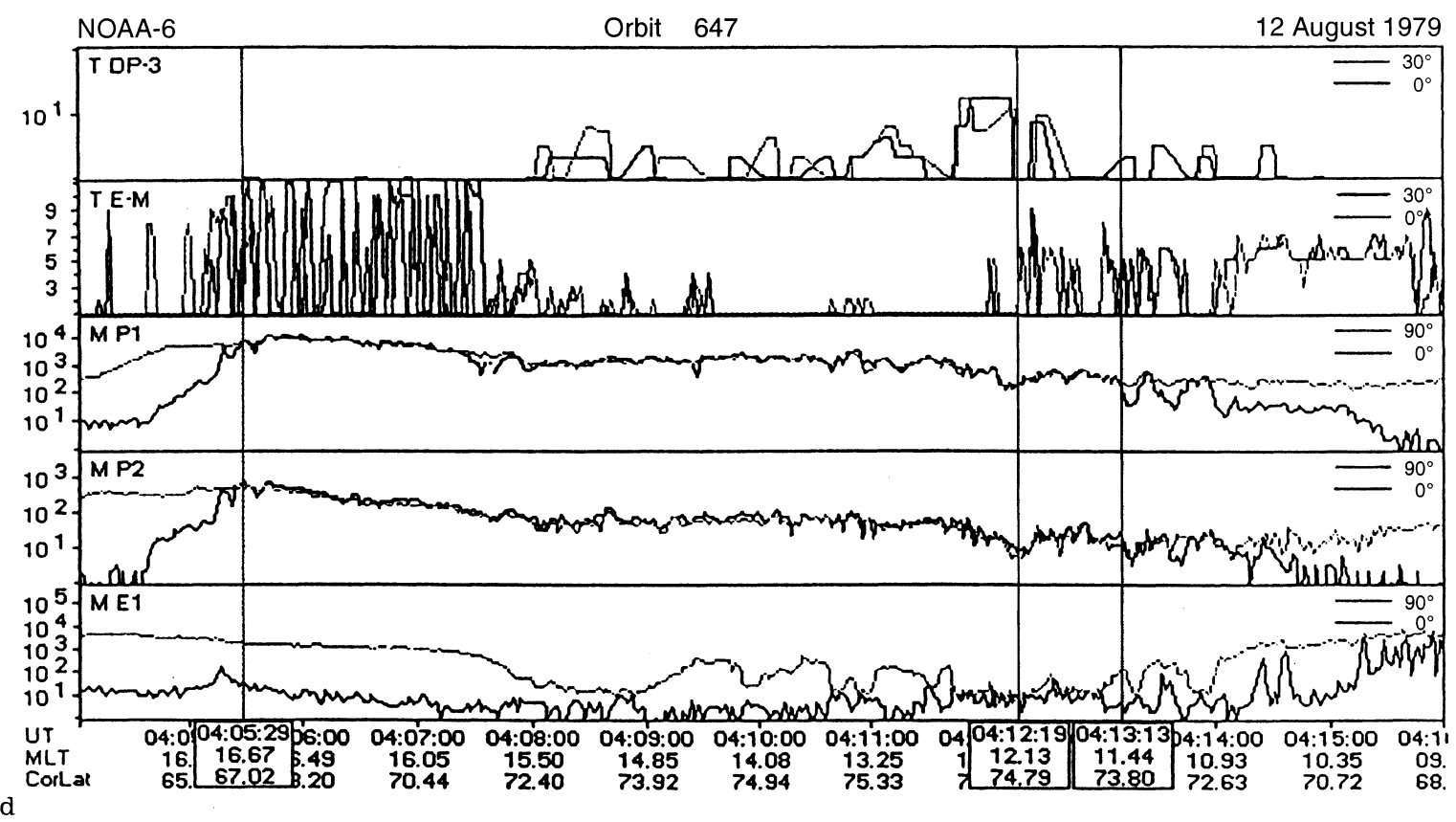




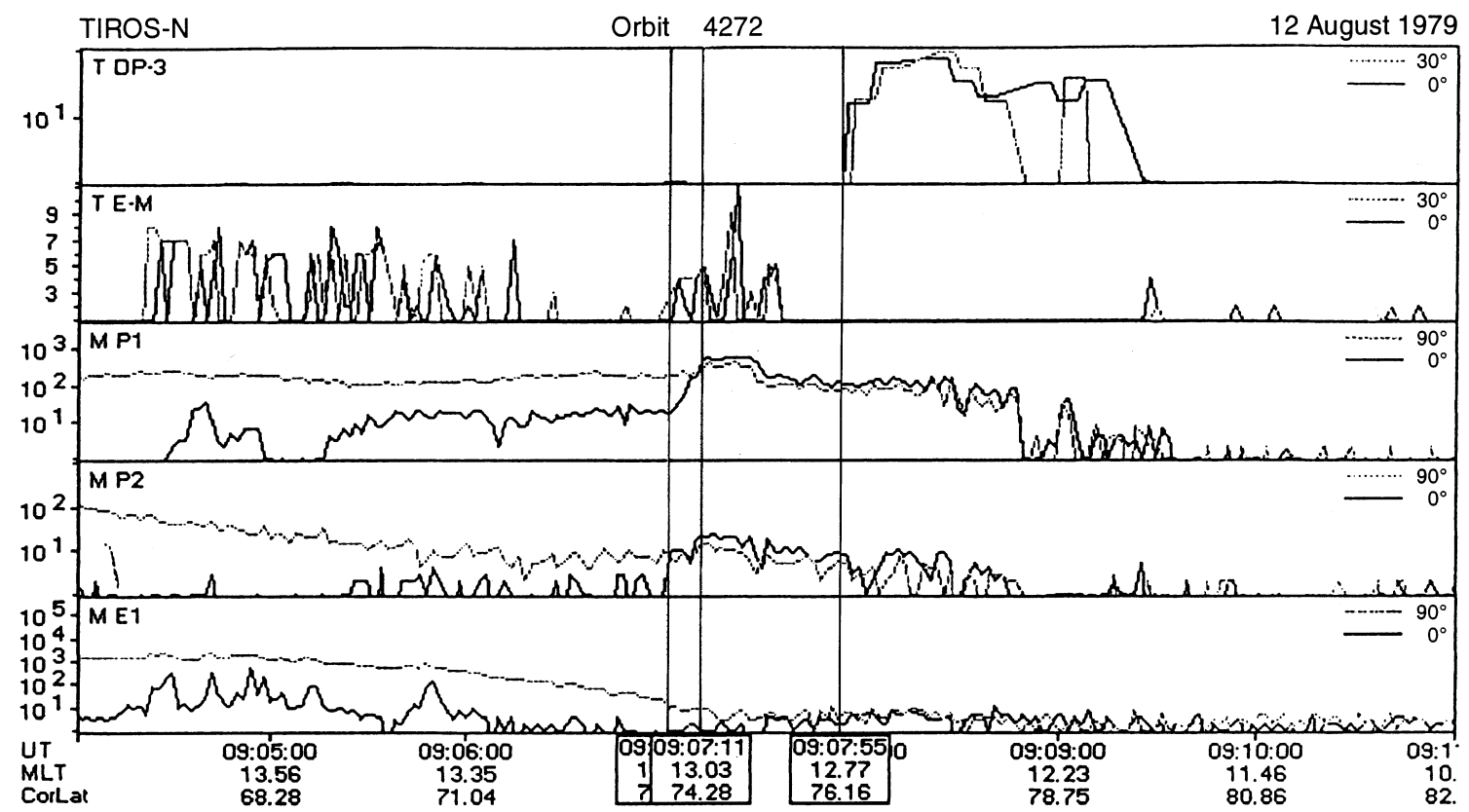

Fig. 7. Same as Fig. 6c, d but for nearly meridional crossing of TIROS spacecraft across the near noon dayside oval

the local time sector between 08 and $16 \mathrm{~h}$ MLT. The isotropic boundaries were determined by using the automatic algorithm described in Sergeev and Gvozdevsky (1995).

The results averaged in $1 / 4$ hour MLT bins are shown in Fig. 8. They do not show the well-defined indentations in question, neither in the behavior of average IB latitude nor in the frequency of the IB registration (real indentation at fixed MLT could result in smaller amount of boundary determination at corresponding MLT). However, by comparing these with model predictions (Fig. 4) two interesting peculiarities may be noticed. One is the obvious shift of the symmetry line of the isotropic boundary by $\sim 1 \mathrm{~h}$ towards on earlier MLT. Another is that the isotropic boundary stays at nearly constant latitude (a plateau-like IB shape) near noon, between $\sim 09$ and $\sim 13 \mathrm{~h}$ MLT. The local time size of this region corresponds to the distance in MLT between the IB indentations in Fig. 4. We shall return to this issue in the Sect. 4. Note that these results were obtained on a reasonably large statistical sample about 100 points in each $1 / 4 \mathrm{~h}$ MLT bin).

We also studied how the shape of isotropic boundary varies depending on the magnetic activity and solar wind parameters. According to physical arguments and previous experience, from a large number of possible geoeffective factors we tested the AE index, the solar wind dynamic pressure $\left(P_{d}\right)$, as well as the $B_{z}$ and $B_{y}$ IMF components. Based on their hourly values, we analyzed corresponding subsets of the IB crossings. We used the least square fitting procedure for each subset to obtain the simplest, cosine-like approximation of the boundary shape in the form

$I B L A T=A_{0}+A_{1} * \cos \left[\pi\left(M L T-M L T_{0}\right) / 12\right]$

To obtain a more accurate shape using limited statistics with noisy data the following modification was used.
Instead of fitting each separate crossing, we used pairs of crossings on the same orbit (at two different MLTs, like

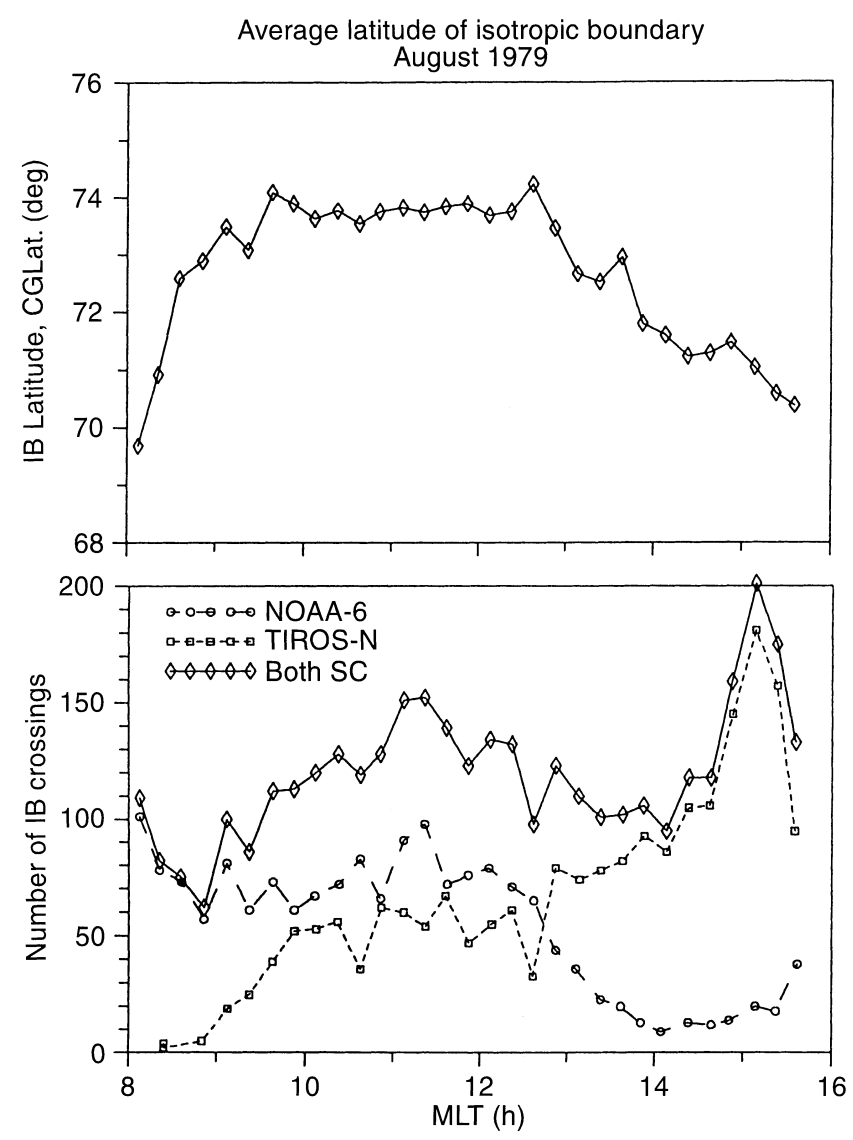

Fig. 8. Statistics of a number of IB crossings near the noon (bottom, including number of crossings by each spacecraft separately as well as the total number of crossings by two spacecraft) and the average behavior of the IB latitude as a function of MLT (top) 
shown in Fig. 6a, b). In that case, the fitting formula (1) is reduced to the formula with only two free parameters (amplitude $A_{1}$ and phase $M L T_{0}$ ). In this way, treating the double crossings as one measurement we determined the least square fit for both the phase and amplitude and then determined the remaining free term $A_{0}$ from the data set. Having in mind the dipole tilt effects (Newell and Meng, 1989), this was done separately for the equinox season (August 1979, TIROS and NOAA6, and April-May 1986, NOAA6, dipole tilt angles DT $\sim 0 \pm 10^{\circ}$ ) and for the winter season (November 1981, NOAA6 and NOAA7, and October 1986, NOAA6 and NOAA10, DT $\sim-20 \pm 5^{\circ}$ ). We also tried different MLT ranges at dayside where the double crossings should occur, however we had a reasonably large amount of data samples for the largest range only between 06 and $18 \mathrm{~h}$ MLT.

The results are given in Table 2, where we present the phase $\left(M L T_{0}\right)$ and the maximal latitude of the isotropic boundary (MLAT $=A_{0}+A_{1}$ ) as well as the average parameters characterizing each subset. These results confirm a dawnside rotation of the symmetry line (phase $M L T_{0}$ is everywhere between 10.5 and $12 \mathrm{~h}$ MLT) in agreement with the average pattern in Fig. 8. The strongest influence comes from the IMF $B_{z}$ component and the AE index: with increasing activity the symmetry line rotates to prenoon sector and the maximal IB latitude decreases. Since $B_{z}$ and $A E$ are known to be well correlated, here we could not determine which of them plays a primary role.

From previous studies (e.g., Newell et al., 1989 and references therein) one would expect to observe a large rotation of the pattern toward earlier (later) MLT when the IMF $B_{y}$ component is negative (positive). However, this was confirmed only in the equinox subset, whereas in winter season the difference between subsets with negative/positive $B_{y}$ is small and has an opposite sense.
One can notice that this difference can be attributed to the difference in average $A E$ (or $B_{z}$ ) in $B_{y}$-dependent subsets. Therefore we conclude that the $B_{y}$-dependent effect, at least, is modest if it exists at all. A more careful study based on a larger statistical sample should be made to distinguish between contributions from IMF $B_{z}$ (external) and $A E$ (internal) highly correlated processes, as well as to study the secondary effects of other parameters.

To characterize quantitatively the energy-dependent spatial dispersion of the isotropic boundary, we processed carefully the near-noon (10-12 MLT) nearmeridional passes for the last 10 days of July 1979 using both TIROS and NOAA6 spacecraft. We always found the IB of $80 \mathrm{keV}$ protons equatorward of the IB of $30 \mathrm{keV}$ protons. The average difference was $0.23 \pm 0.14^{\circ}$ of Corr. Geom. Latitude for 42 passes processed. The corresponding difference predicted by the model computations is $0.2-0.5^{\circ}$ (see Fig. 3 as example).

\subsection{Last closed field line and isotropic boundary at noon meridian}

To compare observations with the modeling results it is important to define experimentally the latitudinal distance between the isotropic boundary and the last closed field line (LCFL), i.e., the (outer) magnetic cusp location. However even the distinction between cusp proper, mantle and LLBL regions is not always clear (e.g., Roeder and Lyons, 1992), and the characterization of the magnetic field topology based on the characteristics of auroral particles is still more doubtful. Instead, we exploit a rare possibility to use the solar electron plateau boundary as the best possible (in our majority opinion) tracer of the LCFL in the dayside auroral zone.

Table 2. Phase $\left(M L T_{0}\right)$ and maximal corr.geom.latitude (MLAT) of the isotropic boundary cosine fit for subsets ordered by interplanetary parameters or activity indices for equinox $(\mathrm{E})$ and winter $(\mathrm{W})$ seasons

\begin{tabular}{|c|c|c|c|c|c|c|c|c|}
\hline \multirow{2}{*}{$\begin{array}{l}\text { Parameter } \\
\text { of subset }\end{array}$} & \multirow[t]{2}{*}{ Season } & \multirow[t]{2}{*}{$\mathrm{NN}$} & \multirow[t]{2}{*}{$M L T_{0}$} & \multirow[t]{2}{*}{ MLAT } & \multicolumn{4}{|c|}{ Average parameters } \\
\hline & & & & & $\begin{array}{l}A E \\
(\mathrm{nT})\end{array}$ & $\begin{array}{l}P_{d} \\
(\mathrm{nPa})\end{array}$ & $\begin{array}{l}B_{z} \\
(\mathrm{nT})\end{array}$ & $\begin{array}{l}B_{y} \\
(\mathrm{nT})\end{array}$ \\
\hline$A E>200 \mathrm{nT}$ & $\mathrm{E}$ & 98 & 11.0 & 73.6 & 411 & 3.3 & -1.5 & -1.4 \\
\hline$A E<100 \mathrm{nT}$ & $\mathrm{E}$ & 118 & 12.0 & 76.4 & 66 & 2.1 & 2.4 & -1.0 \\
\hline$A E>200 \mathrm{nT}$ & $\mathrm{W}$ & 81 & 11.5 & 74.0 & 350 & 3.6 & -0.4 & -1.2 \\
\hline$A E<100 \mathrm{nT}$ & $\mathrm{W}$ & 129 & 12.1 & 75.8 & 54 & 2.7 & 1.7 & -2.0 \\
\hline$B_{z}>2 \mathrm{nT}$ & $\mathrm{E}$ & 66 & 11.8 & 76.2 & 97 & 3.0 & 4.3 & -1.5 \\
\hline$B_{z}<-2 \mathrm{nT}$ & $\mathrm{W}$ & 15 & 10.6 & 73.1 & 330 & 3.6 & -2.7 & 1.1 \\
\hline$B_{z}>2 \mathrm{nT}$ & $\mathrm{W}$ & 31 & 12.1 & 75.7 & 127 & 3.5 & 3.7 & -2.0 \\
\hline$B_{y}<-2 \mathrm{nT}$ & $\mathrm{E}$ & 91 & 10.9 & 75.1 & 231 & 2.7 & 0.8 & -4.9 \\
\hline$B_{y}>2 \mathrm{nT}$ & $\mathrm{E}$ & 50 & 11.6 & 74.6 & 197 & 2.7 & 0.6 & 4.2 \\
\hline$B_{y}<-2 \mathrm{nT}$ & $\mathrm{W}$ & 62 & 11.6 & 75.0 & 176 & 2.9 & 1.5 & -3.4 \\
\hline$B_{y}>2 \mathrm{nT}$ & W & 22 & 11.4 & 74.4 & 265 & 3.8 & -0.7 & 4.1 \\
\hline$P_{d}>4 \mathrm{nPa}$ & $\mathrm{E}$ & 34 & 11.6 & 74.9 & 298 & 6.4 & 1.8 & -3.0 \\
\hline$P_{d}>4 \mathrm{nPa}$ & $\mathrm{W}$ & 26 & 11.3 & 75.4 & 187 & 5.4 & 1.2 & 0.1 \\
\hline$P_{d}<2 \mathrm{nPa}$ & $\mathrm{W}$ & 28 & 12.0 & 74.8 & 104 & 1.5 & 0.3 & -2.3 \\
\hline
\end{tabular}


Here we show the results for intense solar electron event on September 16-17, 1979. This period was characterized by moderate activity (with some moderate substorms on September 16 and mostly quiet on September 17). Figure 9 shows an example of the pass through the dayside and morningside auroral zone with solar electron plateau boundary (SEB) and isotropic boundary marked by the vertical lines. High and very isotropic energetic electron flux continuing at a constant level at high latitudes is the signature of a wellrecognized effect, namely a (rare) solar electron event. At the dayside, its sharp and non-dispersive boundary (SEB) is clearly seen approximately at the poleward edge of energetic electron precipitation (where T-EM panel shows a sharp drop of electron energy down to the lowest value $300 \mathrm{eV})$. The sharp increase of $1 \mathrm{keV}$ proton fluxes (upper panel) occurs here $\sim 0.8^{\circ}$ poleward of the SEB. (In other cases considered we always found SEB poleward of such cusp-like electron signature, but the relationship with $1 \mathrm{keV}$ proton signature was variable. More often, the SEB was placed between electron and proton signatures of equatorward cusp precipitation boundary described already). The ion isotropic boundary, as usual, is at lower latitude, $\sim 2^{\circ}$ equatorward of the SEB. In this event it is clearly seen at $30 \mathrm{keV}$ energy channel whereas in channel $P_{2}$ the signal is variable due to the low count rates.

A summary of locations of isotropic boundaries and solar electron boundaries for two day period of high solar electron fluxes is given in Fig. 10. The pattern splits to two (dayside and nightside) branches with gaps at $\sim 10$ and $\sim 13 \mathrm{~h} \mathrm{MLT}$, but the significance of this fact is questionable due to the limited statistics. However, four cases of very small distance should be noticed between SEB and IB $\left(0.2-0.3^{\circ}\right.$ CGLat), two of them occurred at $\sim 10.5 \mathrm{~h} \mathrm{MLT}$ and another two at 12-12.5 h MLT. However, in the same sector a large separation, about 1 or $2^{\circ}$ CGLat, can also be found showing that this characteristic may be variable (in space or in time). The average distance between the SEB and IB was found to be $1.0 \pm 0.68^{\circ}$ for nine passes between 10 and $12 \mathrm{~h}$ MLT. We shall use this figure later as the estimate of average distance between the isotropic boundary and the last closed field line.

Another feature of Fig. 10 is as follows. In spite of the spread of data points it is clear that SEB latitude has smaller MLT variation than the isotropic boundary has. Accordingly, the distance between the last closed field line and the isotropic boundary increases toward dawn or dusk hours. This parameter also has a large scatter.

\section{Discussion and conclusions}

\subsection{Dayside and nightside sources of dayside isotropic precipitation}

In this study we performed computations using magnetospheric models and analyzed the observations of energetic particles to investigate the characteristics and origin of isotropic proton precipitation in the dayside auroral zone. Our model computations confirmed that a week magnetic field in the outer cusp necessarily should contribute to the regular scattering of energetic protons on closed field lines in $\sim 1-2^{\circ}$ wide zone near the cusp meridian. In a quantitative way, and using existing empirical models, this confirms the previous results of Lyons et al. (1994) and Alem and Delcourt (1995). Our results also extend previous findings by specifying the

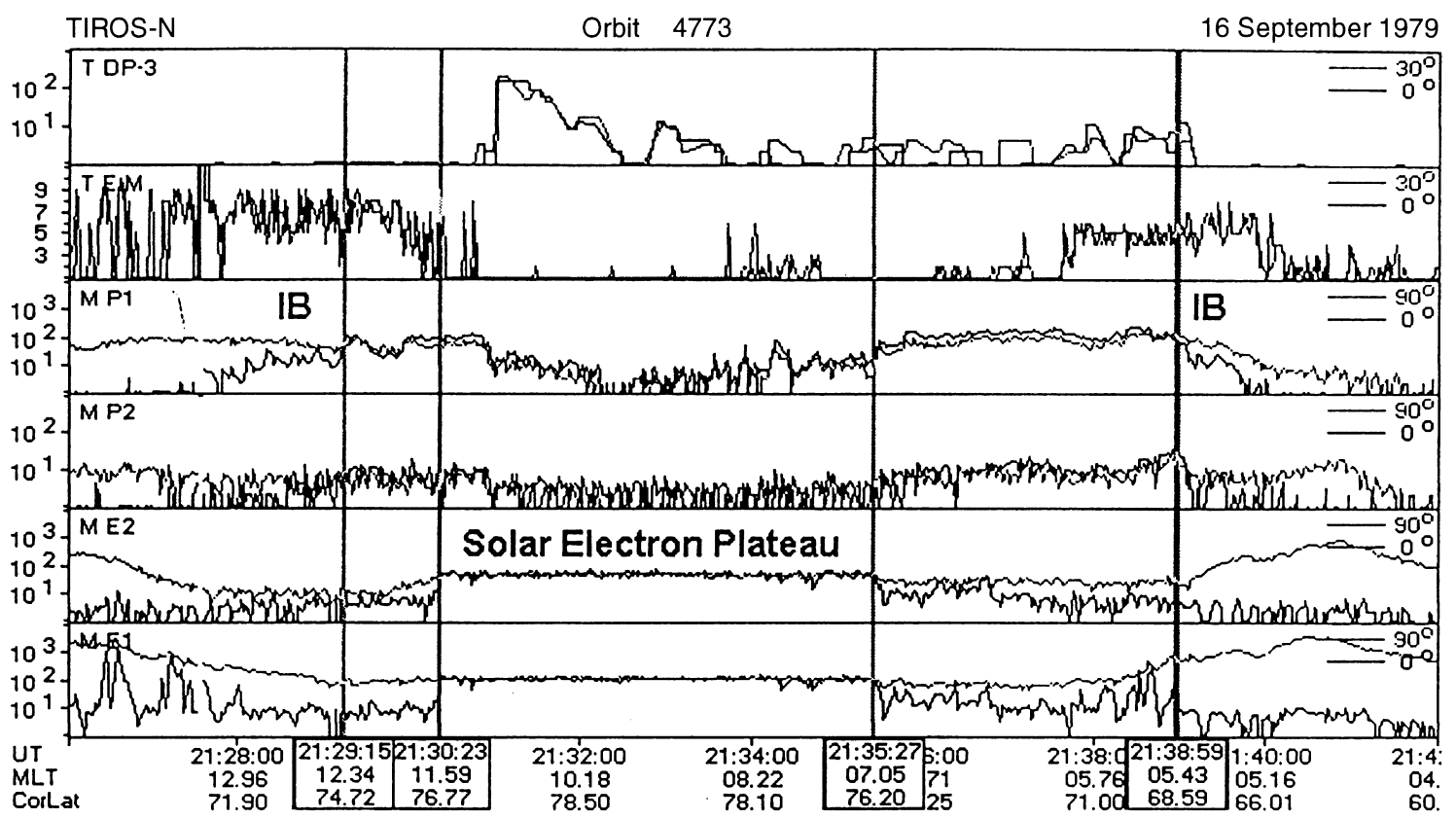

Fig. 9. Same as in Fig. 7 but for the case with large fluxes of energetic solar electrons. To facilitate the visualization of the solar electron flux plateau in the high-latitude region, here we also added data from $>100 \mathrm{keV}$ electron channel $\left(E_{2}\right)$ 
longitudinal range of isotropic precipitation of energetic protons (2-3 h of Local Time centered at noon) produced by this mechanism, as well as providing some other quantitative characteristics such as amount of energy dispersion at the isotropic boundary, equatorward shift of the IB with respect to the last closed field line, etc. These characteristics are in a reasonable quantitative agreement with those found in observations. Therefore, we conclude that isotropic precipitation in the near-noon local time sector is basically provided by the regular scattering process (non-adiabatic pitch angle scattering in the regions of weak and highly-curved magnetic field).

According to our computations, regular scattering on the nightside, near the equatorial magnetopause, is another important contributing process which provides isotropic precipitation observed as close as $\sim 1.5 \mathrm{~h}$ MLT from the noon position. This occurs because of sharp tailward bending of the near-cusp field lines (see Fig. 1). At the local times further from the noon position it joins smoothly the isotropic precipitation from the tail current sheet (e.g., Sergeev et al., 1993). Bearing in mind its origin from nightside equatorial regions, in the later discussion we refer to this part of the modeled isotropic precipitation as the nightside equatorial part (or, the nightside part) although in the ionosphere it may start and end as close to noon as 13.5 and $10.5 \mathrm{~h}$ MLT. (These numbers refer to the model magnetosphere, actually they should be rotated by $\sim 1 \mathrm{~h}$ prenoon to be compared with observations, as discussed later).

\subsection{Symmetry properties of magnetospheric magnetic field following observations}

The real magnetosphere and models may have different symmetry properties. Previous observation-based models (T87, T89), because of the functions employed to describe the external magnetic field, are dawn-dusk symmetric and the latest model T95 (with the exception of interconnection field source) is also only weakly asymmetric. Also, the models are based on the GSM coordinate system which ignores the variable direction of non-radial solar wind, although the authors asked the users to use the properly aberrated coordinate system.

In the real situation, these symmetry properties are different and may change with the time depending on both external and internal factors. First of all, the noonmidnight (GSM) plane in the models should correspond to the aberrated system (to the plane containing the solar wind velocity vector) in the case of observations. The well-known aberration effect due to the Earth's orbital motion gives on average $\sim 4^{\circ}$ aberration which requires a systematic $\sim 0.3 \mathrm{~h}$ MLT shift of the symmetry line toward the earlier MLT. (The individual samples of observed solar wind velocity show the azimuthal deviations from the its average direction as large as $10-20^{\circ}$ on rare occasions. Correspondingly, the actual symmetry line in some occasions may rotate by as much as $\sim 1 \mathrm{~h}$ MLT from its average direction). The empirical results (R. Lundin et al., unpublished manuscript) show that the particle "cusp proper" region is shifted by as much as $\sim 1-2 \mathrm{~h}$ MLT when comparing data sets with positive and negative $V_{y}$ components of the solar wind.

Another external factor could be the longitudinal shifts of the outer magnetic cusp due to the IMF $B_{y}$ dependence of magnetopause reconnection position (e.g., Crooker et al., 1987). However our results show that it is probably a secondary effect.

Our analysis indicates that the amount of rotation (up to $10.5 \mathrm{~h}$ on average during disturbed conditions, see Table 2) is much larger than that expected from the external factors alone. There is some other evidence supporting so large a shift. As an example, we may cite the statistical results by Newell et al. (1989) concerning the cusp latitude as a function of the local time. As follows from their Fig. 4, the highest average cusp latitude is observed at $10.5 \mathrm{~h}$ MLT (with a difference as large as $3-4^{\circ}$ compared to the noon).

As suggested by the strong dependence of the symmetry line azimuth ( $M L T_{0}$ in Table 2$)$ on the $A E$ index and IMF $B_{z}$ component, there may be a stronger internal (magnetospheric) factor distorting the shape of the closed field lines and the mapping. Previously the same amount (and direction) of rotation of the symmetry line was found by Sergeev and Gvozdevsky (1995) who utilized the isotropic boundaries for all MLTs, with some preference for nightside measurements in the data set. As discussed by these authors, the probable explanation is the distortion of the magnetospheric magnetic field. A corresponding distortion (more depressed magnetic field at dusk than at dawn) is consistent with direct spacecraft magnetic measurements in the inner magnetosphere (see discussion and references in this study). A physical reason providing this asymmetry may be the that 'tail-aligned' field-aligned currents are a part of the 'partial ring current' system as known for a long time. (Actually, this field-aligned current may flow on the outer shells of the plasma sheet rather than on dipolelike field lines, see e.g., Crooker and Siscoe, 1981). A more detail discussion on that subject is outside the scope of our work.

These field-aligned currents are not yet (and should be) properly included into the empirical magnetospheric models.

\subsection{Transition between dayside and nightside parts of isotropic precipitation}

Here we comment on apparent disagreement which seem to exist between modeling and observational results concerning the transition between isotropic precipitation from dayside (outer cusp) and nightside regions. In the model computations made for zero dipole tilt angle for a number of models (Fig. 4), both domains still contribute some isotropic precipitation on close field lines at 10.5 and $13.5 \mathrm{~h}$ MLT meridians. However, at $\sim 30$ to $100 \mathrm{keV}$ proton energies its width is very small, about a few tenths of a degree of latitude, which is comparable to both the tracing accuracy of the 
last closed field line and spatial resolution of observations used. In this region we may expect to detect virtually no isotropic precipitation on the closed field lines. With a shift of only about $1 / 2 \mathrm{~h}$ in magnetic local time (Fig. 4), the model predicts rapidly increasing width of isotropic precipitation. Therefore, according to the model predictions, that part of isotropic precipitation zone of $30-80 \mathrm{keV}$ protons located on the closed field lines should consist of two separate belts, dayside and nightside, separated by narrow gaps.

Some limited support for this possibility comes from a summary of relative locations of the solar electron plateau boundaries with isotropic boundaries plotted in Fig. 10. In particular in some passes the boundaries (SEB and IB) could be detected very close $\left(\sim 0.2^{\circ}\right)$ to each other, suggesting a very narrow zone of isotropic precipitation on closed field lines. However, at other UT times, a wide isotropic zone could be observed at the same MLT. Also, in Fig. 6 we showed an example of observations of the high fluxes of energetic electrons in the isotropic proton precipitation zone which continued from dusk to dawn. Therefore, this effect (very narrow zone of isotropic protons) is certainly not as systematic a feature as suggested by models.

In Fig. 11 the modeled and average observed IB positions are plotted together. Assuming that rotation of the symmetry line to the prenoon sector is a real feature not yet included into the magnetospheric models (as discussed already) we rotated modeling results shown in Fig. 4 by $1 \mathrm{~h}$ in local time. Also, taking into account the fact that average distance between the last closed field line and isotropic boundary near the noon is $1^{\circ}$ (Sect. 3.3), we refered the average observed IB latitude (Fig. 8) with respect to the LCFL at noon. In this way we plotted the observation and modeling results in the same format in Fig. 11. It now shows that a 3-4 h MLT-wide plateau part of the isotropic boundary centered at the 'cusp footpoint' (which is now at $11 \mathrm{~h} \mathrm{MLT}$ ) reasonably reproduces the dayside part of modeled isotropic precipitation. However, the observa-

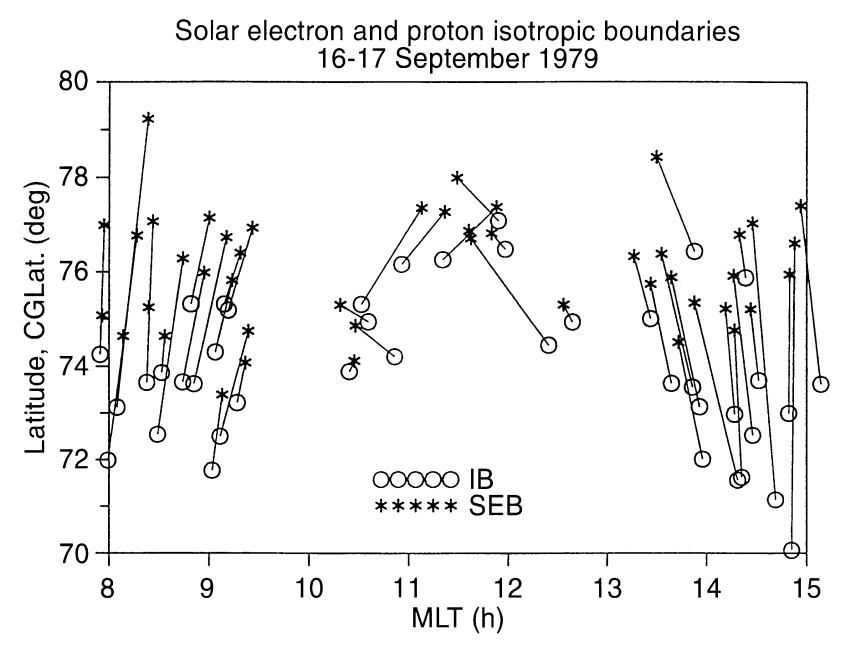

Fig. 10. Statistics of SEB and IB locations during solar electron event on September 16-17, 1979 tions deviate from model predictions (1) by the absence of poleward kinks of the boundary (in the gap between dayside and nightside sources of dayside precipitation, discussed above), and (2) by a considerably faster decrease of the IB latitude with a distance from the symmetry line $(\sim 3 \mathrm{~h}$ MLT from that line the observed IB latitude is already $2-4^{\circ}$ lower than predicted). While feature (1) could be, to some extent, explained by the shifts of the symmetry line, dipole tilt effects (shifts of the gap in the local time) or by other temporal variations which may smooth sharp features in the longitudinal distribution, feature (2) certainly shows a large quantitative difference. We attribute this difference to the defects of magnetospheric models in describing the magnetic field structure in such a complicated regions as the near-cusp domain and the LLBL domain are.

\subsection{Concluding remarks}

After finding the regions of regular scattering (nonadiabatic effects of particle motion in the regular magnetospheric magnetic field) in the magnetospheric models and comparing predictions with observations we arrive at the following conclusions.

1. Important characteristics of isotropic proton precipitation on dayside (the width of proton isotropic precipitation on the closed field lines, local time dependence and energy dispersion of the IB) are reasonably explained by the magnetospheric models. This suggests that isotropic precipitation on the dayside is also caused

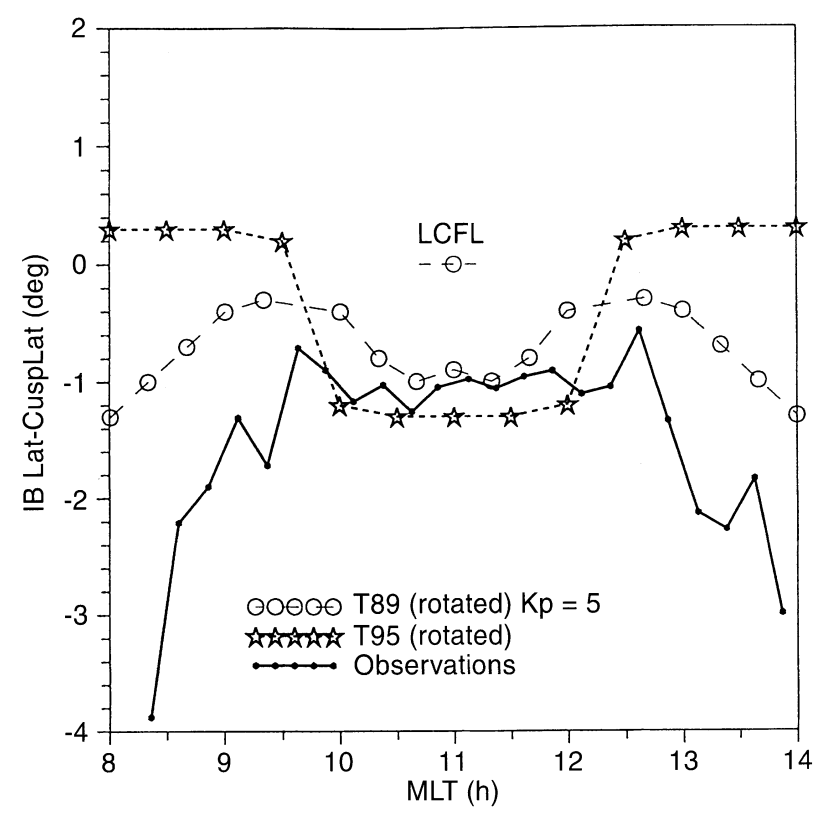

Fig. 11. Same as Fig. 4, but with overlapping modeled isotropic boundaries (for zero dipole tilt) and average observed isotropic boundary. Modeled IBs are here shifted by $1 \mathrm{~h}$ MLT toward earlier time to facilitate comparison with observations. The observed IB is taken from Fig. 8 but the expected latitude of the last closed field line at noon is subtracted (distance between LCFL and IB was taken to be $1^{\circ}$ CGLatitude according to the results of Sect. 3.3) 
by the regular particle scattering in the magnetospheric magnetic field. Also this mechanism nicely explains the continuous existence of the isotropic precipitation on closed field lines under any activity conditions and for any solar wind parameters.

2. Quantitative comparison of predictions and observations reveals some important quantitative differences, including: (1) no clear gaps between precipitation regions (on closed field lines) produced in the outer cusp and near the nightside magnetopause; (2) dawndusk asymmetry of the isotropic boundary in MLT, with lower latitudes on the dusk side and the overall rotation of the symmetry line toward dawn (which depends mostly on IMF $B_{z}$ and $A E$ ); (3) the observed MLT variation of the IB latitude is larger than in any model tested (however predictions of different models vary considerably amongst themselves).

After confirming that proton isotropic precipitation on the dayside is controlled by the magnetospheric magnetic field, we interpret these differences as indications of potential problems with existing magnetospheric models and mapping based on them in this complicated dayside domain near the cusp. Also, we expect that the comparison with observed isotropic boundaries will be an independent test for future magnetospheric models.

Acknowledgements. The data of NOAA-type spacecraft were provided through the National Geophysical Data Center in Boulder. We thank Boris Gvozdevsky for providing us with a software package for processing and visualization of NOAA particle data. The work by VAS and GRB was supported partly by the International Science Foundation (ISF grant NWT-300) and partly by the RFBR (RFBR grant 96-05-64019). Work by PTN was funded by NSF Grant ATM-9531767 (GEM).

Topical Editor K. H. Glaßmeier thanks R. L. Lyons and another referee for their help in evaluating this paper.

\section{References}

Alem, F. and D. Delcourt, Nonadiabatic precipitation of ions at the cusp equatorward edge, J. Geophys. Res, 100, 19321-19328, 1995.

Carbary, J. F. and C.-I. Meng, Correlation of cusp latitude with $B_{z}$ and AE(12) using nearly one year's data, J. Geophys. Res., 91, 10047-10054, 1986.
Crooker, N. U., and G. L. Siscoe, Birkeland currents as the cause of the low-latitude asymmetric disturbance field, J. Geophys. Res., 86, 11201-11210, 1981.

Crooker, N. U., J. Berchem, and C. T. Russell, Cusp displacement at the magnetopause for large IMF y-component, J. Geophys. Res., 92, 13467-13471,1987.

Delcourt, D. C., J. A. Sauvaud, R. F. Martin, and T. E. Moore, On the nonadiabatic precipitation of ions from the near-Earth plasma sheet, J. Geophys. Res., 101, 17409-17418, 1996.

Hauge, R., and F. Soraas, Precipitation of $>115 \mathrm{keV}$ protons in the evening and forenoon sectors in relation to the magnetic activity, Planet. Space Sci., 23, 1141-1154, 1975.

Hill, V. J., D. S. Evans, and H. H. Sauer, TIROS/NOAA satellites space environment monitor. Archive tape documentation. NOAA technical memorandum ERL SEL-71. Boulder, Colorado. 1985.

Kaufmann, R. L., D. J. Larson, P. Beidl, and C. Lu, Mapping and energization in the magnetotail, 1. Magnetospheric boundaries, J. Geophys. Res., 98, 9307-9320, 1993.

Lundblad, J. A., F. Soraas, and K. Aarsnes, Substorm morphology of $>100 \mathrm{keV}$ protons, Planet. Space Sci., 27, 841-865, 1979.

Lyons, L. R., M. Schulz, D. C. Pridmore-Brown, and J. L. Roeder, Low-latitude boundary layer near noon: an open field line model, J. Geophys. Res., 99, 17,367-17,377, 1994.

Newell, P. T., and C. I. Meng, Dipole tilt angle effects on the latitude of the cusp and cleft/LLBL, J. Geophys. Res., 94, 69496953, 1989.

Newell, P. T., C. I. Meng, D. G. Sibeck, and R. P. Lepping, Some low-altitude cusp dependencies on the interplanetary magnetic field, J. Geophys. Res., 94, 8921-8927, 1989.

Roeder, J. L., and L. R. Lyons, Energetic and magnetosheath energy particle signatures of the LLBL at low altitude near noon, J. Geophys. Res., 97, 13,817-13,828, 1992.

Sergeev V. A., and B. B. Gvozdevsky, MT-index - a possible new index to characterize the magnetic configuration of magnetotail, Ann. Geophysicae, 13, 1093-1103, 1995.

Sergeev, V. A., M. V. Malkov, and K. Mursula, Testing of the isotropic boundary algorithm method to evaluate the magnetic field configuration in the tail, J. Geophys. Res., 98, 7609-7620, 1993.

Tsyganenko, N. A., Global quantitive models of the geomagnetic field in the cislunar magnetospheric for different disturbance levels., Planet. Space Sci., 35, 1347-1358, 1987.

Tsyganenko, N. A. A magnetospheric magnetic field model with a warped tail current sheet, Planet. Space Sci., 37, 1, 5-20, 1989.

Tsyganenko, N. A. Modelling the Earth's magnetospheric magnetic field confined within a realistic magnetopause, J. Geophys. Res., 100, 5599-5612, 1996. 